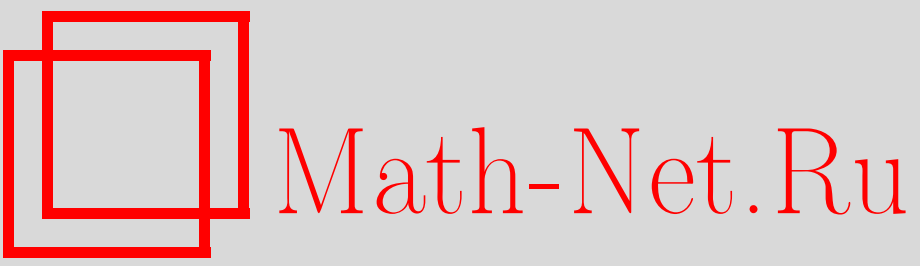

А. Э. Ковальский, Г. П. Пронько, $Q$-операторы Бакстера для интегрируемой DST-цепочки, TMФ, 2005, том 142, номер 2, 310-321

DOI: https://doi.org/10.4213/tmf1784

Использование Общероссийского математического портала Math-Net.Ru подразумевает, что вы прочитали и согласны с пользовательским соглашением

http://www.mathnet.ru/rus/agreement

Параметры загрузки:

IP: 54.147 .182 .235

26 апреля 2023 г., 11:10:32

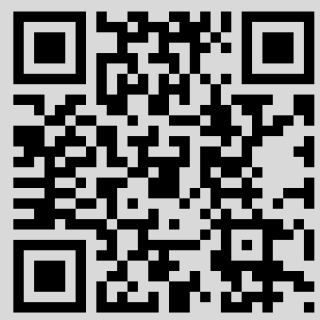


ТЕОРЕТИЧЕСКАЯ

И МАТЕМАТИЧЕСКАЯ

ФИЗИКА

Том 142, № 2

февраль, 2005

(C) 2005 г.

А. Э. Ковальский* ${ }^{*}$ Г. П. Пронько ${ }^{\dagger}$

\section{$Q$-ОПЕРАТОРЫ БАКСТЕРА ДЛЯ ИНТЕГРИРУЕМОЙ DST-ЦЕПОЧКИ}

Построены операторы Бакстера для интегрируемой DST-цепочки в виде следа от монодромий некоторых $M$-операторов, которые действуют в квантовом и вспомогательном пространствах. В рамках этой процедуры получены два базисных $M$-оператора и установлены некоторые функциональные соотношения между ними, такие как переплетающие соотношения и соотношения вронскианного типа между двумя базисными $Q$-операторами.

Ключевые слова: интегрируемые цепочки, алгебраический анзац Бете, функциональные уравнения.

\section{1. ВВЕДЕНИЕ}

Интегрируемая периодическая квантовая DST-цепочка ${ }^{1)}$ является квантовой системой, гамильтониан для которой мы выберем в следуюшем виде (он соответствует определенному выбору параметров $\omega_{0}, \gamma, \epsilon$ и $m_{i j}$ в гамильтониане из работы [1]):

$$
H=\sum_{k=1}^{N}\left[\varphi_{k}^{+} \varphi_{k}+\frac{\left(\varphi_{k}^{+} \varphi_{k}\right)^{2}}{2}+\varphi_{k+1}^{+} \varphi_{k}\right]
$$

где канонические переменные $\varphi_{k}^{+}$и $\varphi_{k}$ удовлетворяют коммутационным соотношениям $\left[\varphi_{i}, \varphi_{j}^{+}\right]=\delta_{i j}$ и периодическим условиям $\varphi_{k+N}=\varphi_{k}, \varphi_{k+N}^{+}=\varphi_{k}^{+}$. Эта система может быть рассмотрена в рамках квантового метода обратной задачи рассеяния или метода $R$-матрицы. С квантовой DST-цепочкой связан оператор Лакса, действующий в тензорном произведении $n$-го квантового пространства и двумерного вспомогательного пространства $\mathbb{C}^{2}$ (см. [2], [3]), вида

$$
L_{n}(x)=\left(\begin{array}{cc}
x-i / 2-i \varphi_{n}^{+} \varphi_{n} & \varphi_{n}^{+} \\
\varphi_{n} & i
\end{array}\right),
$$

\footnotetext{
${ }^{1)} \mathrm{DST}$-цепочка (discrete self-trapping) - цепочка с дискретным самозахватом.
}

*ГНЦ ИФВЭ, Протвино, Московская обл., Россия; МФТИ, г. Долгопрудный, Московская обл., Россия.

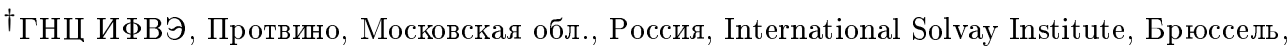
Бельгия. E-mail: pronko@mx.ihep.su 
где $x$ - спектральный параметр. При этом фундаментальные коммутационные соотношения для элементов оператора Лакса могут быть записаны в следующей $R$-матричной форме:

$$
R_{12}(x-y) L_{n}^{1}(x) L_{n}^{2}(y)=L_{n}^{2}(y) L_{n}^{1}(x) R_{12}(x-y),
$$

где индексы 1 и 2 обозначают различные вспомогательные пространства,

$$
R_{12}(x)=x+i P_{12}
$$

$P_{12}$ - оператор перестановки во вспомогательных пространствах 1 и 2 . Такие же коммутационные соотношения верны и для матришы монодромии $T(x)=\prod_{n=1}^{N} L_{n}(x)$ (множители упорядочены справа налево), и это, в свою очередь, означает, что $[t(x), t(y)]=0$, где $t(x)=\operatorname{Tr} T(x)$ (в этом выражении след берется по вспомогательному пространству). Коэффициенты полинома $t(x)$ являются, таким образом, семейством коммутирующих операторов, причем гамильтониан $H$ и оператор числа частищ принадлежат этому семейству (а именно, если $t(x)=\sum_{k=0}^{N}(x-i / 2)^{k} H^{(k)}$, то оператор числа частиц $\hat{n}=\sum_{k=1}^{N} \varphi_{k}^{+} \varphi_{k}=i H^{(N-1)}$, гамильтониан $\left.H=i H^{(N-1)}-\left(H^{(N-1)}\right)^{2} / 2+H^{(N-2)}\right)$.

Собственные векторы и собственные значения оператора $t(x)$ могут быть построены с помощью алгебраического анзаца Бете (далее ААБ, см., например, [4]). При этом подходе рассматривается матрица монодромии

$$
T(x)=\prod_{n=1}^{N} L_{n}(x)=\left(\begin{array}{ll}
\widehat{A}(x) & \widehat{B}(x) \\
\widehat{C}(x) & \widehat{D}(x)
\end{array}\right)
$$

Для DST-модели существует так называемый вакуум Бете $C(x) \Omega=0$ (здесь $\Omega=$ $\prod \otimes \omega_{k}$, где $\left.\varphi_{k} \omega_{k}=0\right)$. Векторы $\widehat{B}\left(x_{1}\right) \ldots \widehat{B}\left(x_{l}\right) \Omega$ являются собственными векторами оператора $\operatorname{Tr} T(x)=\widehat{A}(x)+\widehat{D}(x)$ с собственными значениями

$$
t(x)=\left(x-\frac{i}{2}\right)^{N} \prod_{j=1}^{l} \frac{\left(x-x_{j}+i\right)}{\left(x-x_{j}\right)}+i^{N} \prod_{j=1}^{l} \frac{\left(x-x_{j}-i\right)}{\left(x-x_{j}\right)}
$$

причем $x_{i}$ подчиняются уравнениям Бете

$$
\prod_{j=1}^{l} \frac{\left(x_{i}-x_{j}-i\right)}{\left(x_{i}-x_{j}+i\right)}=-\frac{\left(x_{i}-i / 2\right)^{N}}{i^{N}}
$$

Таким образом, полиномы $q(x)=\prod_{j=1}^{l}\left(x-x_{j}\right)$ удовлетворяют уравнению Бакстера

$$
t(x) q(x)=\left(x-\frac{i}{2}\right)^{N} q(x-i)+i^{N} q(x+i) .
$$

Следуя Бакстеру [5], определим оператор $Q(x)$ с помошью следуюшего соотношения:

$$
t(x) Q(x)=\left(x-\frac{i}{2}\right)^{N} Q(x-i)+i^{N} Q(x+i)
$$


при этом $[t(x), Q(y)]=0,[Q(x), Q(y)]=0$.

Рассматриваемая модель занимает промежуточное место между двумя другими интегрируемыми моделями: спиновой $X X X$-цепочкой и цепочкой Тоды (операторы Лакса этих моделей также переплетаются рациональной $R$-матрицей (4)). Как и в случае спиновой XXX-цепочки, для DST-модели сушествует $Q$-оператор с полиномиальными собственными значениями по спектральному параметру. Он соответствует ААБ. Если мы рассмотрим уравнение (5) как дискретный аналог дифференциального уравнения, сразу возникнет вопрос о сушествовании второго решения уравнения (5). Вторые решения интенсивно изучались в последнее время [6], [7]. Собственные значения второго $Q$-оператора для DST-модели являются мероморфными функциями (в случае цепочки Тоды нельзя построить ААБ, однако существует два $Q$-оператора: один с целыми собственными значениями, другой с мероморфными [8]). В следуюшей части эти два решения, удовлетворяюшие (6), будут построены.

Сушествование второго $Q$-оператора, линейно независимого от первого, видно из следуюших простых соображений (аналогичные рассуждения для случая спиновой $X X X$ цепочки см. в [7]). Рассмотрим уравнение Бакстера для собственного значения первого $Q$-оператора $q(x)$, которое является полиномом степени $n$ (в случае DST-цепочки $n$ равно числу частиц, находящихся на узлах, т.е. собственному значению оператора числа частиц $\left.\sum_{i=1}^{N} \varphi_{i}^{+} \varphi_{i}\right)$, и собственного значения следа матрицы монодромии $t(x)$, являюшегося полиномом степени $N$. Имеем

$$
t(x) q(x)=\left(x-\frac{i}{2}\right)^{N} q(x-i)+i^{N} q(x+i)
$$

или

$$
\frac{t(x)}{q(x+i) q(x-i)}=\frac{(x-i / 2)^{N}}{q(x) q(x+i)}+\frac{i^{N}}{q(x) q(x-i)} .
$$

Умножим это соотношение на $\Gamma^{N}(-i(x-i / 2))$, получим

$$
\frac{t(x) \Gamma^{N}(-i(x-i / 2))}{q(x+i) q(x-i)}=\frac{i^{N} \Gamma^{N}(-i(x+i / 2))}{q(x) q(x+i)}+\frac{i^{N} \Gamma^{N}(-i(x-i / 2))}{q(x) q(x-i)} .
$$

Введем обозначение

$$
S(x)=\frac{i^{N} \Gamma^{N}(-i(x+i / 2))}{q(x) q(x+i)}
$$

тогда

$$
\frac{t(x) \Gamma^{N}(-i(x-i / 2))}{q(x+i) q(x-i)}=S(x)-S(x-i) .
$$

Перепишем $S(x)$ в виде

$$
S(x)=i^{N} \Gamma^{N}\left(-i\left(x+\frac{i}{2}\right)\right)\left[\frac{q_{1}(x)}{q(x+i)}+\frac{q_{2}(x)}{q(x)}\right]
$$


где $q_{1}(x)$ и $q_{2}(x)$ - полиномы степени, меньшей $n$. Подставим это разложение в уравнение Бакстера (8):

$$
\frac{t(x)}{q(x+i) q(x-i)}=\left(x-\frac{i}{2}\right)^{N}\left[\frac{q_{1}(x)}{q(x+i)}+\frac{q_{2}(x)}{q(x)}\right]+i^{N}\left[\frac{q_{1}(x-i)}{q(x)}+\frac{q_{2}(x-i)}{q(x-i)}\right] .
$$

Так как $t(x)$ - полином, то $(x-i / 2)^{N} q_{2}(x)+i^{N} q_{1}(x-i)=r(x) q(x)$, где $r(x)$ - полином степени, меньшей $N$. Выразив функцию $q_{1}(x)$ через $q_{2}(x)$ и $r(x)$, подставим ее в $S(x)$, получим

$$
\begin{aligned}
S(x)= & i^{N} \Gamma^{N}\left(-i\left(x+\frac{i}{2}\right)\right) r(x+i)+i^{N} \Gamma^{N}\left(-i\left(x+\frac{i}{2}\right)\right) \frac{q_{2}(x)}{q(x)}- \\
& -\Gamma^{N}\left(-i\left(x+\frac{3 i}{2}\right)\right) \frac{q_{2}(x+i)}{q(x+i)}
\end{aligned}
$$

Теперь наша задача состоит в том, чтобы представить $S(x)$ в виде

$$
S(x)=\frac{p(x+i)}{q(x+i)}-\frac{p(x)}{q(x)}
$$

тогда $p(x)$ будет искомым собственным значением второго $Q$-оператора. Действительно, из (10) получаем

$$
i^{N} \Gamma^{N}\left(-i\left(x+\frac{i}{2}\right)\right)=p(x+i) q(x)-p(x) q(x+i)
$$

и $i^{N} \Gamma^{N}(-i(x-i / 2))=p(x) q(x-i)-p(x-i) q(x)$. Умножая последнее равенство на $(-i(x-i / 2))^{N}$ и вычитая из предыдущего, мы видим, что $p(x)$ удовлетворяет тому же уравнению Бакстера, что и $q(x)$ :

$$
t(x) p(x)=\left(x-\frac{i}{2}\right)^{N} p(x-i)+i^{N} p(x+i) .
$$

Видно, что необходимо найти такую функцию $g(x)$, что

$$
g(x+i)-g(x)=i^{N} \Gamma^{N}\left(-i\left(x+\frac{i}{2}\right)\right) r(x+i) .
$$

Будем искать $g(x)$ в виде

$$
g(x)=\sum_{k=0}^{\infty} f(-i x-k)
$$

тогда $g(x+i)-g(x)=-f(-i x)$, т.е. $f(-i x)=-i^{N} \Gamma^{N}(-i(x+i / 2)) r(x+i)$, и

$$
g(x)=-i^{N} \sum_{k=0}^{\infty} \Gamma^{N}\left(-i\left(x+\frac{i}{2}\right)-k\right) r(x+i-i k) .
$$


Искомое собственное значение есть

$$
p(x)=g(x) q(x)-i^{N} \Gamma^{N}\left(-i\left(x+\frac{i}{2}\right)\right) q_{2}(x) .
$$

Оно является мероморфной функцией от $x$ с полюсами при целых значениях $y=-i x+$ $1 / 2$ (сходимость ряда для $g(x)$ при нецелом $-i(x+i / 2)$ обеспечивается слагаемым $-k$ в аргументе гамма-функции).

В качестве иллюстрации рассмотрим простой пример полиномиального решения уравнения Бакстера в случае двух степеней свободы

$$
q(x)=x^{2}-2 i x+\frac{1}{4}
$$

Это решение соответствует собственному вектору $\frac{1}{\sqrt{2}}(|2,0\rangle-|0,2\rangle)$ и собственному значению матрицы монодромии

$$
t(x)=x^{2}-3 i x-\frac{9}{4}
$$

Здесь введено следующее обозначение для векторов квантового пространства:

$$
\left|k_{1}, k_{2}\right\rangle=\left(\varphi_{1}^{+}\right)^{k_{1}}\left(\varphi_{2}^{+}\right)^{k_{2}}|0,0\rangle
$$

где $|0,0\rangle$ - бетевский вакуум (т.е. $\left.\varphi_{1}|0,0\rangle=\varphi_{2}|0,0\rangle=0\right)$.

Явное построение полиномов $q_{1}, q_{2}, r$ по методу, описанному выше, дает

$$
q_{1}(x)=-\frac{i}{2} x+\frac{1}{4}, \quad q_{2}(x)=\frac{i}{2} x+\frac{3}{4}, \quad r(x)=\frac{i}{2} x+\frac{1}{4} .
$$

При этом для собственного значения второго $Q$-оператора получаем

$$
\begin{aligned}
p(x)= & \left(x^{2}-2 i x+\frac{1}{4}\right) \sum_{k=0}^{\infty} \Gamma^{2}\left(-i x+\frac{1}{2}-k\right)\left(\frac{i}{2} x+\frac{k}{2}-\frac{1}{4}\right)+ \\
& +\Gamma^{2}\left(-i x+\frac{1}{2}\right)\left(\frac{i}{2} x+\frac{3}{4}\right) .
\end{aligned}
$$

\section{2. БАЗИСНЫЕ $Q$-ОПЕРАТОРЫ ДЛЯ DST-МОДЕЛИ}

Будем искать базисные $Q$-операторы с помошью метода, описанного в [8]. Согласно этому методу $Q^{(1,2)}$-операторы суть следы операторов $\widehat{Q}^{(1,2)}$, которые представляют собой произведения $M^{(1,2)_{n}}$-операторов, действующих в $n$-м квантовом пространстве и во вспомогательном пространстве Г (пространстве представления алгебры Гейзенберга $\left.\left[\rho, \rho^{+}\right]=1\right)$. Так как мы будем рассматривать произведение $L(x) M^{(1,2)}(x)$, то общим вспомогательным пространством для этого объекта будет $\Gamma \otimes \mathbb{C}^{2}$, где мы можем ввести проекторы

$$
\Pi_{i j}^{+}=\left(\begin{array}{c}
1 \\
\rho
\end{array}\right)_{i} \frac{1}{\left(\rho^{+} \rho+1\right)}\left(1, \rho^{+}\right)_{j}, \quad \Pi_{i j}^{-}=\left(\begin{array}{c}
-\rho^{+} \\
1
\end{array}\right)_{i} \frac{1}{\left(\rho^{+} \rho+2\right)}(-\rho, 1)_{j} .
$$


Следуя [9], мы налагаем на произведения $L(x) M^{(1,2)}(x)$ и $M^{(1,2)}(x) L(x)$ условие, что они являются треугольными в смысле проекторов $\Pi^{ \pm}$: для $M_{n}^{(1)}(x)$ справедливы равенства

$$
\begin{aligned}
& \Pi_{i k}^{-}\left(L_{n}(x)\right)_{k l} M_{n}^{(1)}(x) \Pi_{l j}^{+}=0 \\
& \Pi_{i k}^{+} M_{n}^{(1)}(x)\left(L_{n}(x)\right)_{k l} \Pi_{l j}^{-}=0
\end{aligned}
$$

для $M_{n}^{(2)}(x)$ справедливы равенства

$$
\begin{aligned}
& \Pi_{i k}^{+}\left(L_{n}(x)\right)_{k l} M_{n}^{(2)}(x) \Pi_{l j}^{-}=0, \\
& \Pi_{i k}^{-} M_{n}^{(2)}(x)\left(L_{n}(x)\right)_{k l} \Pi_{l j}^{+}=0 .
\end{aligned}
$$

Рассмотрим систему уравнений для $M^{(1)}$. Из первого уравнения в $(23)$ следует, что

$$
\begin{aligned}
M^{(1)}(x)\left(\begin{array}{l}
1 \\
\rho
\end{array}\right)_{i} & =\tilde{L}(x)_{i j}\left(\begin{array}{l}
1 \\
\rho
\end{array}\right)_{j} A^{(1)}(x), \\
B^{(1)}(x)\left(\begin{array}{l}
1 \\
\rho
\end{array}\right)_{i} & =\tilde{L}(x+i)_{i j}\left(\begin{array}{l}
1 \\
\rho
\end{array}\right)_{j} M^{(1)}(x),
\end{aligned}
$$

где мы ввели оператор

$$
\tilde{L}(x)=\left(\begin{array}{cc}
i & -\varphi^{+} \\
-\varphi & x-3 i / 2-\varphi^{+} \varphi
\end{array}\right),
$$

который обладает следуюшими свойствами: $L(x) \tilde{L}(x)=i(x-i / 2) I(I-$ единичная матрица), $L(x)+\tilde{L}(x+i)=\operatorname{Tr} L(x) \cdot I$ (это тождество обеспечивает сдвиг аргумента в (25), что и приводит в итоге к уравнению в конечных разностях). Система (25) допускает решение в виде $B^{(1)}(x)=c M^{(1)}(x+i), A^{(1)}(x)=c^{-1} M^{(1)}(x)$, где $c$ - число. Выберем $c=i$. Рассматривая аналогично условие треугольности для правого умножения (второе из уравнений (23)), мы приходим к системе

$$
\begin{aligned}
& \tilde{L}(x+i)_{i j}\left(\begin{array}{c}
1 \\
\rho
\end{array}\right)_{j} M^{(1)}(x)=M^{(1)}(x+i)\left(\begin{array}{l}
1 \\
\rho
\end{array}\right)_{i}, \\
& M^{(1)}(x) L(x)_{i j}\left(\begin{array}{c}
-\rho^{+} \\
1
\end{array}\right)_{j}=i\left(\begin{array}{c}
-\rho^{+} \\
1
\end{array}\right)_{i} M^{(1)}(x+i) .
\end{aligned}
$$

Такое же рассмотрение для $M^{(2)}$ приводит к системе

$$
\begin{gathered}
\tilde{L}(x+i)_{i j}\left(\begin{array}{c}
-\rho^{+} \\
1
\end{array}\right)_{j} M^{(2)}(x)=M^{(2)}(x+i)\left(\begin{array}{c}
-\rho^{+} \\
1
\end{array}\right)_{i}, \\
M^{(2)}(x) L(x)_{i j}\left(\begin{array}{l}
1 \\
\rho
\end{array}\right)_{j}=i\left(\begin{array}{l}
1 \\
\rho
\end{array}\right)_{i} M^{(2)}(x+i) .
\end{gathered}
$$

При этом верны следующие правила умножения:

$$
\left(L_{n}(x)\right)_{i j} M_{n}^{(1)}(x)=\left(\begin{array}{c}
1 \\
\rho
\end{array}\right)_{i} M_{n}^{(1)}(x-i) \frac{1}{\rho^{+} \rho+1}\left(1, \rho^{+}\right)_{j}+
$$




$$
\begin{aligned}
& +\left(\begin{array}{c}
-\rho^{+} \\
1
\end{array}\right)_{i} \frac{1}{\rho^{+} \rho+2} M_{n}^{(1)}(x+i)(-\rho, 1)_{j}+\Pi_{i k}^{+}\left(L_{n}(x)\right)_{k l} M_{n}^{(1)}(x) \Pi_{l j}^{-} ; \\
& \left(L_{n}(x)\right)_{i j} M_{n}^{(2)}(x)=\left(\begin{array}{c}
1 \\
\rho
\end{array}\right)_{i} \frac{1}{\rho^{+} \rho+1} M_{n}^{(2)}(x+i)\left(1, \rho^{+}\right)_{j}+ \\
& \quad+\left(\begin{array}{c}
-\rho^{+} \\
1
\end{array}\right)_{i} M_{n}^{(2)}(x-i) \frac{1}{\rho^{+} \rho+2}(-\rho, 1)_{j}+\Pi_{i k}^{-}\left(L_{n}(x)\right)_{k l} M_{n}^{(2)}(x) \Pi_{l j}^{+} ; \\
& M_{n}^{(1)}(x)\left(L_{n}(x)\right)_{i j}=\left(\begin{array}{c}
1 \\
\rho
\end{array}\right)_{i} \frac{1}{\rho^{+} \rho+1} M_{n}^{(1)}(x-i)\left(1, \rho^{+}\right)_{j}+ \\
& \quad+\left(\begin{array}{c}
-\rho^{+} \\
1
\end{array}\right)_{i} M_{n}^{(1)}(x+i) \frac{1}{\rho^{+} \rho+2}(-\rho, 1)_{j}+\Pi_{i k}^{-}\left(L_{n}(x)\right)_{k l} M_{n}^{(1)}(x) \Pi_{l j}^{+} ; \\
& M_{n}^{(2)}(x)\left(L_{n}(x)\right)_{i j}=\left(\begin{array}{c}
1 \\
\rho
\end{array}\right)_{i} M_{n}^{(2)}(x+i) \frac{1}{\rho^{+} \rho+1}\left(1, \rho^{+}\right)_{j}+ \\
& \quad+\left(\begin{array}{c}
-\rho^{+} \\
1
\end{array}\right)_{i} \frac{1}{\rho^{+} \rho+2} M_{n}^{(2)}(x-i)(-\rho, 1)_{j}+\Pi_{i k}^{+}\left(L_{n}(x)\right)_{k l} M_{n}^{(2)}(x) \Pi_{l j}^{-} .
\end{aligned}
$$

Мы не рассматриваем структуры последних членов в правых частях этих правил умножения. Действительно, треугольная структура (23), (24) будет верна также и для произведений $L_{n}$ и $M_{n}$, так как квантовые операторы с разными $n$ коммутируют друг с другом. Поэтому эти соотношения гарантируют, что следы монодромий $M^{(1,2)}$ по вспомогательному пространству (если они существуют),

$$
Q^{(1,2)}(x)=\operatorname{Tr} \widehat{Q}^{(1,2)}(x)=\operatorname{Tr} \prod_{k=1}^{N} M_{k}^{(1,2)}(x),
$$

удовлетворяют уравнению Бакстера (6).

Для решения систем (26), (27) мы будем использовать голоморфное представление операторов $\rho, \rho^{+}$. При этом оператор $\rho^{+}$становится оператором умножения $\left(\rho^{+} \psi\right)(\alpha)=$ $\alpha \psi(\alpha)$, а оператор $\rho$ - оператором дифференцирования: $(\rho \psi)(\alpha)=\partial \psi(\alpha) / \partial \alpha$. Действие оператора в голоморфном представлении определяется его ядром:

$$
(\widehat{M} \psi)(\alpha)=\int d^{2} \mu(\beta) M(\alpha, \bar{\beta}) \psi(\beta),
$$

где мера интегрирования определена следуюшим образом: $d^{2} \mu(\beta)=e^{-\beta \bar{\beta}} d \beta d \bar{\beta}$. В этом представлении операторы, удовлетворяющие системам (26), (27), вьплядят следуюшим образом:

$$
\begin{aligned}
& M^{(1)}(x, \alpha, \bar{\beta})=e^{-i \bar{\beta} \varphi^{+}} \frac{\Gamma(-i(x-i / 2))}{\Gamma\left(-\varphi^{+} \varphi-i(x-i / 2)\right)} e^{-i \alpha \varphi}, \\
& M^{(2)}(x, \alpha, \bar{\beta})=e^{i \alpha \varphi} e^{i \pi \varphi^{+} \varphi} \Gamma\left(-\varphi^{+} \varphi-i(x-i / 2)\right) e^{i \bar{\beta} \varphi^{+}} .
\end{aligned}
$$

Для нахождения монодромии $\widehat{Q}^{(1,2)}(x, \alpha, \bar{\beta})$ необходимо взять упорядоченное произведение $M^{(1,2)}$-операторов:

$$
\begin{aligned}
\widehat{Q}^{(i)}(x, \alpha, \bar{\beta})= & \int \prod_{i=1}^{N-1} d^{2} \mu\left(\gamma_{i}\right) M_{N}^{(i)}\left(x, \alpha, \bar{\gamma}_{N-1}\right) M_{N-1}^{(i)}\left(x, \gamma_{N-1}, \bar{\gamma}_{N-2}\right) \times \cdots \\
& \cdots \times M_{2}^{(i)}\left(x, \gamma_{2}, \bar{\gamma}_{1}\right) M_{1}^{(i)}\left(x, \gamma_{1}, \bar{\beta}\right) .
\end{aligned}
$$


Для нахождения $Q^{(1,2)}$-операторов необходимо взять след операторов $\widehat{Q}^{(1,2)}$. След оператора $Q$ в голоморфном представлении имеет вид

$$
\operatorname{Tr} Q=\int d^{2} \mu(\alpha) \widehat{Q}(\alpha, \bar{\alpha})
$$

где $\widehat{Q}(\alpha, \bar{\alpha})$ - ядро оператора $\widehat{Q}$.

Собственные значения $Q^{(1)}(x)$ являются полиномами по $x$. В самом деле, $Q^{(1)}$ действует на базисные векторы $\left|n_{1}, n_{2}, \ldots, n_{N}\right\rangle=\left(\varphi_{1}^{+}\right)^{n_{1}}\left(\varphi_{2}^{+}\right)^{n_{2}} \ldots\left(\varphi_{N}^{+}\right)^{n_{N}}|0\rangle$, где $|0\rangle-$ бетевский вакуум, $\varphi_{k}|0\rangle=0, k=1, \ldots, N$, следуюшим образом:

$$
\begin{aligned}
& Q^{(1)}(x)\left|n_{1}, \ldots, n_{N}\right\rangle=\sum_{m_{1}, \ldots, m_{N}}^{n_{1}, \ldots, n_{N}} \prod_{k=1}^{N} \frac{(-1)^{m_{k}}}{m_{k} !} \times \\
& \quad \times \frac{\Gamma(-i(x-i / 2))}{\Gamma\left(-i(x-i / 2)-n_{k}+m_{k}\right)} \frac{n_{k} !}{\left(n_{k}-m_{k}\right) !}\left|\ldots, n_{k}-m_{k}+m_{k-1}, \ldots\right\rangle .
\end{aligned}
$$

Отсюда видно, что $Q^{(1)}(x)$ оставляет инвариантным подпространство векторов с одинаковым общим числом частиц $n=n_{1}+n_{2}+\cdots+n_{N}$, и все матричные элементы $Q^{(1)}$ являются полиномами по $x$. В дальнейшем будет доказано, что $\left[Q^{(1)}\left(x_{1}\right), Q^{(1)}\left(x_{2}\right)\right]=0$, поэтому собственные значения операторов $Q^{(1)}$ являются полиномами по $x$. Построенньй в работе [3] $Q$-оператор соответствует $Q^{(1)}$. Его действие на базисные векторы (в [3] выбрано координатное представление для квантовых операторов, в котором базисными векторами являются полиномы $x_{1}^{n_{1}} \ldots x_{n}^{n_{N}}$ ) схоже с действием $Q^{(1)}$ в $(37)$.

Для сравнения приведем действие оператора $Q^{(2)}$ на те же базисные векторы:

$$
\begin{aligned}
& Q^{(2)}(x)\left|n_{1}, \ldots, n_{N}\right\rangle=e^{i \pi N} \sum_{m_{1}, \ldots, m_{N}=0}^{\infty} \prod_{k=1}^{N} \Gamma\left(-i x-1 / 2-n_{k}-m_{k-1}\right) \times \\
& \quad \times \frac{\left(m_{k-1}+n_{k}\right) !}{m_{k} !\left(n_{k}+m_{k-1}-m_{k}\right) !}\left|\ldots, n_{k}+m_{k-1}-m_{k}, \ldots\right\rangle .
\end{aligned}
$$

В отличие от первого оператора здесь суммирование ведется по бесконечному набору $m_{k}$, который, однако, ограничен условиями $m_{k}-m_{k-1} \leqslant n_{k}$.

Отметим, что при выборе некоторых реализаций для квантовых операторов и операторов вспомогательного пространства возможна факторизация $Q$-операторов, впервые рассмотренная в работе [10] (см. также [9]). Например, в координатном представлении для квантовых операторов и операторов во вспомогательном пространстве ядро $Q$-оператора имеет следующий вид (см. [3], [9]):

$$
Q\left(x_{1}, \ldots, x_{N}, x_{1}^{\prime}, \ldots, x_{N}^{\prime}\right)=\prod_{k=1}^{N} q_{k}\left(x_{k}, x_{k+1}^{\prime}, x_{k}^{\prime}\right)
$$

В работе [3] построен один из $Q$-операторов в форме (39). В подобной факторизованной форме можно также построить второй $Q$-оператор (это было отмечено в [3]). Однако с точки зрения подхода работы [8] происхождение такой факторизации неясно. 
В простейшем случае $N=1$ получаем $\left(n=\varphi^{+} \varphi\right)$

$$
\begin{aligned}
Q^{(1)}(x) & =\sum_{k=0}^{n} \frac{n !}{k !(n-k) !} \frac{\Gamma(-i x+1 / 2)}{\Gamma(-i x+1 / 2-n-k)}, \\
Q^{(2)}(x) & =e^{i \pi n} \sum_{m=0}^{\infty} \frac{(n+m) !}{m ! n !} \Gamma(-i x-1 / 2-n-m) .
\end{aligned}
$$

Как и ожидалось, собственные значения $Q^{(1)}$ являются полиномами степени $n$, а собственные значения $Q^{(2)}$ являются мероморфными функциями спектрального параметра.

Можно найти решения систем (26), (27) в следуюшем виде:

$$
\begin{aligned}
& M^{(1)}(x, \rho)=\mathrm{P}^{\rho \varphi}\left(i-\varphi \rho^{+}\right)^{-i(x-i / 2)} e^{-x \pi / 2}, \\
& M^{(2)}(x, \rho)=\Pi^{\rho \varphi} \Gamma\left(-\rho^{+} \rho-\varphi^{+} \varphi-i(x-i / 2)\right),
\end{aligned}
$$

где оператор $\mathrm{P}^{\rho \varphi}=e^{\pi / 2\left(\varphi^{+} \rho-\varphi \rho^{+}\right)} e^{i \pi / 2\left(\rho^{+} \rho-\varphi^{+} \varphi\right)}$ обладает свойствами оператора перестановки:

$$
\begin{array}{ll}
\mathrm{P}^{\rho \varphi} \varphi=i \rho \mathrm{P}^{\rho \varphi}, & \mathrm{P}^{\rho \varphi} \varphi^{+}=-i \rho^{+} \mathrm{P}^{\rho \varphi}, \\
\varphi \mathrm{P}^{\rho \varphi}=-i \mathrm{P}^{\rho \varphi} \rho, & \varphi \mathrm{P}^{\rho \varphi^{+}}=i \mathrm{P}^{\rho \varphi} \rho^{+},
\end{array}
$$

$\Pi^{\rho \varphi}$ - оператор со следуюшими свойствами: $\varphi \Pi^{\rho \varphi}=-i \rho \Pi^{\rho \varphi}, \Pi^{\rho \varphi} \varphi^{+}=-i \Pi^{\rho \varphi} \rho^{+}$. При этом, однако, $\varphi^{+} \Pi^{\rho, \varphi}=i\left[\Pi^{\rho, \varphi}, \rho^{+}\right], \Pi^{\rho, \varphi} \varphi=-i\left[\Pi^{\rho, \varphi}, \rho\right]$. Явное выражение для оператора $\Pi^{\rho, \varphi}$ выглядит следуюшим образом:

$$
\begin{aligned}
\Pi^{\rho \varphi}= & {\left[1+\sum_{k=1}\left(i \varphi \rho^{+}\right)^{k} \frac{\Gamma\left(\rho^{+} \rho+1\right)}{\Gamma\left(\rho^{+} \rho+k+1\right)}+\sum_{k=1}\left(i \varphi^{+} \rho\right)^{k} \frac{\Gamma\left(\varphi^{+} \varphi+1\right)}{\Gamma\left(\varphi^{+} \varphi+k+1\right)}\right] \times } \\
& \times \frac{\Gamma\left(\rho^{+} \rho+\varphi^{+} \varphi+1\right)}{\Gamma\left(\rho^{+} \rho+1\right) \Gamma\left(\varphi^{+} \varphi+1\right)} e^{i \pi \varphi^{+} \varphi} .
\end{aligned}
$$

Операторы $M_{n}^{(1,2)}$ и $L_{n}(x)$ удовлетворяют определенным переплетаюшим соотношениям, из которых следует, что $Q$-операторы и трансфер-матрица коммутируют. Так как метод получения перелетаюших соотношений такой же, как и в случае цепочки Тоды, более того, все переплетаюшие $R$-матрицы совпадают с переплетаюшими матрицами для цепочки Тоды, то ниже мы приводим все переплетаюшие соотношения без их вывода. Подробности можно найти в работе [8].

Для оператора Лакса и $M$-операторов справедливо равенство

$$
R_{k l}^{(i)}(x-y)\left(L_{n}(x)\right)_{l m} M_{n}^{(i)}(y)=M_{n}^{(i)}(y)\left(L_{n}(x)\right)_{k l} R_{l m}^{(i)}(x-y) .
$$

Соответствуюшие $R$-матришы имеют вид

$$
\begin{aligned}
& R_{k l}^{(1)}(x-y)=\left(\begin{array}{cc}
x-y+i \rho^{+} \rho & -i \rho^{+} \\
-i \rho & i
\end{array}\right), \\
& R_{k l}^{(2)}(x-y)=\left(\begin{array}{cc}
i & i \rho^{+} \\
i \rho & x-y+i+i \rho^{+} \rho
\end{array}\right) .
\end{aligned}
$$


Отметим, что в работе [3] уравнение (45) для матрицы $R^{(1)}$ было рассмотрено как определяюшее для элементарных блоков, из которых потом строится матрица монодромии, след которой есть $Q$-оператор.

Для операторов $M^{(1)}$ и $M^{(2)}$, действуюших в разных вспомогательных пространствах, верно соотношение

$$
M_{n}^{(1)}(x, \rho) M_{n}^{(2)}(y, \tau) R^{12}(x-y)=R^{12}(x-y) M_{n}^{(2)}(y, \tau) M_{n}^{(1)}(x, \rho),
$$

где переплетаюшая матрища $R^{12}$ задана в виде ядра в голоморфном представлении,

$$
R^{12}(x, \alpha, \bar{\beta} ; \gamma, \bar{\delta})=\sum_{n=0} \frac{((\alpha-\gamma)(\bar{\beta}-\bar{\delta}))^{n}}{n ! \Gamma(-i x+n+1)}
$$

Здесь $\alpha, \bar{\beta}$ - голоморфиные переменные для вспомогательного пространства представления операторов $\rho$ и $\rho^{+}$, и $\gamma, \bar{\delta}$ - голоморфные переменные для вспомогательного пространства представления операторов $\tau$ и $\tau^{+}$.

Для $M^{(1)}$-операторов, действующих в различных вспомогательных пространствах, имеем

$$
R^{(11)}(x-y) M^{(1)}(x, \rho) M^{(1)}(y, \tau)=M^{(1)}(y, \tau) M^{(1)}(x, \rho) R^{(11)}(x-y),
$$

где

$$
R^{(11)}(x)=P_{\rho \tau}\left(1+\rho^{+} \tau\right)^{-i x},
$$

$P_{\rho \tau}-$ оператор перестановки во вспомогательных пространствах.

Для $M^{(2)}$-операторов, действующих в различных вспомогательных пространствах, справедливо соотношение

$$
R^{(22)}(x-y) M^{(2)}(x, \rho) M^{(2)}(y, \tau)=M^{(2)}(y, \tau) M^{(2)}(x, \rho) R^{(22)}(x-y),
$$

где

$$
R^{(22)}(x)=P_{\rho \tau}\left(1+\tau^{+} \rho\right)^{-i x} .
$$

Эти переплетаюшие соотношения приводят к взаимной коммутации $Q$-операторов и $t$-матрицы:

$$
\left[t(x), Q^{(i)}(y)\right]=0, \quad\left[Q^{(i)}(x), Q^{(j)}(y)\right]=0, \quad i, j=1,2 .
$$

Итак, нами построено два решения операторного уравнения Бакстера. Докажем теперь линейную независимость этих решений, определив однопараметрическое семейство конечноразностных аналогов определителя Вронского

$$
W_{m}=Q_{1}(x-i m) Q_{2}(x+i)-Q_{1}(x+i) Q_{2}(x-i m),
$$


где $m$ - неотрицательное целое число. Рассмотрим свойства этих объектов, вытекаюшие непосредственно из уравнения Бакстера:

$$
\begin{aligned}
& t(x) Q_{1}(x)=\left(x-\frac{i}{2}\right)^{N} Q_{1}(x-i)+i^{N} Q_{1}(x+i), \\
& t(x) Q_{2}(x)=\left(x-\frac{i}{2}\right)^{N} Q_{2}(x-i)+i^{N} Q_{2}(x+i) .
\end{aligned}
$$

Умножим первое уравнение на $Q_{2}(x)$, второе - на $Q_{1}(x)$ и вычтем одно уравнение из другого, получим

$$
\left(x-\frac{i}{2}\right)^{N} W_{0}(x-i)=i^{N} W_{0}(x),
$$

т.е. $W_{0}$ обязательно содержит множитель $\Gamma^{N}(-i(x+i / 2))$. Далее, умножим первое из уравнений $(56)$ на $Q_{2}(x-i)$, второе - на $Q_{1}(x-i)$. Получим

$$
W_{1}(x)=(-i)^{N} t(x) W_{0}(x-i) .
$$

В случае произвольного $m$, умножая первое уравнение на $Q_{2}(x-i m)$, второе - на $Q_{1}(x-$ $i m)$, получаем

$$
\left(x-\frac{i}{2}\right)^{N} W_{m-2}(x-2 i)+i^{N} W_{m}(x)=t(x) W_{m-1}(x-i) .
$$

Из этих уравнений видно, что можно найти все $W_{m}$, если известно $W_{0}$. Конечно, все эти рассуждения имеют смысл при наличии двух решений уравнения Бакстера, если $W_{0} \neq 0$ тождественно (как раз такие и были построены в данной работе). Рекуррентным соотношениям вида (57) удовлетворяют собственные значения трансфер-матриш $t_{l}(x)$ (матриц монодромии во вспомогательном пространстве представления спина $l=$ $m / 2)$. Семейство $t_{l}$ можно получить, используя следующее выражение для операторов Лакса во вспомогательных пространствах со спинами $l$ :

$$
L_{l}(x)=i^{2 l} e^{-i l^{+} \varphi^{+}} \frac{\Gamma\left(l^{3}-i x\right)}{\Gamma(-i x-l)} e^{-i l^{-} \varphi} .
$$

Здесь операторы $l^{k}$ - операторы спина $l$, множитель $i^{2 l} \Gamma^{-1}(-i(x+i / 2))$ введен для того, чтобы при $l=0$ и $l=1$ получались, соответственно, $L_{0}=1$ и $L_{1 / 2}(x)=L(x)(L(x)-$ оператор Лакса (2)). Из соотношения (16) видно, что

$$
W_{0}(x)=i^{N} \Gamma^{N}(-i(x+i / 2)) .
$$

Явное вычисление $W_{0}$ для решений, построенных методом, описанным в работе [8], дает

$$
W_{0}(x)=e^{i \pi \hat{n}} \Gamma^{N}(-i(x+i / 2)),
$$

где $\hat{n}$ - оператор полного числа частиц. Для этой пары, таким образом, получаем, что

$$
W_{m}(x)=e^{i \pi \hat{n}}(-i)^{2 l N} \Gamma^{N}(-i x-1 / 2) t_{l}(x),
$$

или

$$
Q_{1}(x-i m) Q_{2}(x+i)-Q_{1}(x+i) Q_{2}(x-i m)=e^{i \pi \hat{n}}(-i)^{2 l N} \Gamma^{N}(-i x-1 / 2) t_{l}(x) .
$$




\section{3. ЗАКЛЮЧЕНИЕ}

В нашей работе мы построили базисные $Q$-операторы в форме следов монодромий базисных локальных $M$-операторов для случая интегрируемой DST-модели. $Q$-операторы представлены в виде рядов по каноническим операторам $\varphi_{k}, \varphi_{k}^{+}$с хорошо определенным действием на векторы квантового пространства. Получены переплетающие соотношения, означаюшие, что $Q$-операторы и след матрицы монодромии $t$ коммутируют. Получены функциональные соотношения вронскианного типа, показьваюшие линейную независимость $Q$-операторов и связь между $Q$-операторами и трансфер-матрицами во вспомогательных пространствах представлений высших спинов.

Отметим некоторые нерешенные задачи. Было бы интересно построить $Q$-операторы для небольшого числа степеней свободы как функции семейства коммутируюших операторов, связанных с $t(x)$. Неясным остается происхождение факторизаций $Q$-операторов, возникаюших в некоторых представлениях [3], [9].

Метод, описанный в данной работе, позволяет найти $M$-операторы в наиболее интересном случае спиновой $X X X$-цепочки (они совпадают с операторами Лакса $L(x)$ и $\tilde{L}(x)$, если поменять местами вспомогательное и квантовое пространство), но при этом следы их монодромий расходятся. Однако сушествует процедура построения $Q$-операторов, аналогичная описанной в [9], для случая спиновой $X X X \mathrm{SL}(2, \mathbb{C})$-цепочки [11]. Таким образом, случай спиновой $X X X$-цепочки заслуживает дальнейшего исследования.

Благодарности. Работа вьполнена при поддержке грантов РФФИ № 00-15-96645, № 01-02-16585, CRDF MO-011-0, Министерства образования E00-3.3-62 и INTAS 0000561.

\section{Список литературы}

[1] A. C. Scott, J. C. Eilbeck. Phys. Lett. A. 1986. V. 119. P. 60-64.

[2] V.Z. Enolskii, M. Salerno, N.A. Kostov, A.C. Scott. Physica Scripta. 1991. V. 43. P. 229-235.

[3] V. B. Kuznetsov, M. Salerno, E. K. Sklyanin. J. Phys. A. 2000. V. 33. P. 171-189.

[4] L. D. Faddeev. How algebraic Bethe Anzatz works for intergable models. In: Quantum Symmetries/Symmetries Quantiques. Proc. of the Les Houches Summer School, Session LXIV, Les Houches, France, August 1 - September 8, 1995. Eds. A. Connes, K. Gawedski, J. Zinn-Justin. Amsterdam: North-Holland, 1998. P. 149; hep-th/9605187.

[5] R. J. Baxter. Stud. Appl. Math. 1971. P. L51-69; Ann. Phys. N. Y. 1972. V. 70. P. 193-228; 1973. V. 76. P. 1-71.

[6] V. V. Bazhanov, S. L. Lukyanov, A. B. Zamolodchikov. Commun. Math. Phys. 1997. V. 190. P. $247-78 ; 1998$. V. 200. P. 297-324.

[7] G.P. Pronko, Yu. G. Stroganov. J. Phys. A. 1999. V. 32. P. 2333-2340; hep-th/9808153.

[8] G. P. Pronko. J. Phys. A33. 2000. V. 33. P. 8251-8266; nlin.SI/0003002.

[9] V. V. Bazhanov, Yu. G. Stroganov. J. Statist. Phys. 1990. V. 59. P. 799-817.

[10] V. Pasquer, M. Gaudin. J. Phys. A. 1992. V. 25. P. 5243-5252.

[11] S. E. Derkachov. J. Phys. A. 1999. V. 32. P. 5299-5316; solv-int/9902015. 